\title{
Narrow band imaging hysteroscopy: A comparative study using randomized video images
}

\author{
IORI KISU $^{1}$, KOUJI BANNO ${ }^{1}$, YUSUKE KOBAYASHI ${ }^{1}$, ASUKA ONO ${ }^{1}$, KENTA MASUDA ${ }^{1}$, \\ ARISA UEKI $^{1}$, HIROYUKI NOMURA ${ }^{1}$, AKIRA HIRASAWA ${ }^{1}$, TAKAYUKI ABE ${ }^{2}$, \\ KEISUKE KOUYAMA $^{2}$, NOBUYUKI SUSUMU ${ }^{1}$ and DAISUKE AOKI ${ }^{1}$ \\ ${ }^{1}$ Department of Obstetrics and Gynecology, ${ }^{2}$ The Center for Clinical Research, \\ School of Medicine, Keio University, Tokyo, Japan
}

Received May 24, 2011; Accepted June 20, 2011

DOI: 10.3892/ijo.2011.1131

\begin{abstract}
Narrow band imaging (NBI) has been used in the gastrointestinal endoscopy field as a novel endoscopic imaging technique and has contributed to improved qualitative diagnosis and detection of lesions. However, there are only a few studies of use of NBI in the gynecology field. We applied NBI in flexible hysteroscopy at our outpatient clinic and evaluated the utility of NBI hysteroscopy for diagnosis of malignant endometrial lesions by comparison of the sensitivity and specificity between white light alone and white light+NBI using hysteroscopic video images. The subjects were 65 patients with a suspected endometrial lesion in the uterine cavity. These patients underwent flexible hysteroscopy using NBI in addition to conventional white light. Video images from 65 patients were edited into two groups, white light alone (WL group) and white light+NBI (NBI group) (130 images in total). Computerized block randomization of the order was then performed. Four raters independently diagnosed the images without use of other clinical information. Using the pathological diagnosis as the gold standard, we evaluated the sensitivity and specificity of diagnosis of atypical endometrial hyperplasia (AEH) or endometrial carcinoma compared between the WL and NBI groups. The sensitivity of diagnosis of AEH or endometrial carcinoma was numerically higher in the NBI group for all raters, and the average sensitivity was significantly higher in the NBI group compared to the WL group (78.6\% vs. 63.7\%, $\mathrm{P}<0.001)$. The specificity for each rater and the average specificity were comparable between the two groups. Compared to white light hysteroscopy, NBI hysteroscopy had a higher sensitivity for diagnosis of AEH or endometrial carcinoma without loss of specificity. This suggests that NBI hysteroscopy may be more
\end{abstract}

Correspondence to: Dr Kouji Banno, Department of Obstetrics and Gynecology, School of Medicine, Keio University, 35 Shinanomachi, Shinjuku-ku, Tokyo 160-8582, Japan

E-mail: kbanno@sc.itc.keio.ac.jp

Key words: narrow band imaging, hysteroscopy, malignant endometrial lesions useful than white light hysteroscopy for endoscopic diagnosis of malignant endometrial lesions.

\section{Introduction}

In hysteroscopy, the uterine cavity is directly observed using an endoscope. This method is used as a gynecological examination of the uterine cavity in patients with suspected endometrial lesions. Hysteroscopy is indicated for abnormalities detected in transvaginal ultrasonography and endometrial cytohistology, and in cases with metrorrhagia, hypermenorrhea, prolongation of menstruation, and infertility. Hysteroscopy has been suggested to be a superior examination for investigation of the uterine cavity $(1,2)$, and high diagnostic accuracies for abnormalities in the uterine cavity (3) and endometrial carcinoma have been achieved in studies comparing hysteroscopy and histology $(4,5)$. In contrast, Lasmar et al found sensitivities for diagnoses of endometrial hyperplasia (EH) and carcinoma using white light hysteroscopy of 56.3 and $80.0 \%$, respectively, and suggested that this relatively poor performance of hysteroscopy may be due to a lack of standard diagnostic criteria based on morphological changes of endometrial lesions (6). Pathological examination of a biopsy specimen is recommended for diagnosis of endometrial lesions, and particularly lesions in the uterine cavity, but this requires use of thick operative sheaths and cervical dilation, with accompanying pain and risks.

Narrow band imaging (NBI) is a novel endoscopic imaging technique in which images of micro-blood vessels in the mucosal superficial layer and mucosal surface are enhanced by changing the spectrum of observation light to narrow bands. NBI has been used in digestive endoscopy and has improved qualitative diagnosis and detection of lesions (7). In NBI, the light spectrum is shifted to wavelengths of $415 \pm 30$ and $540 \pm 30 \mathrm{~nm}$, and wavelength-induced changes in visibility are utilized. The absorption spectrum of circulating oxidized hemoglobin has peaks at 415 and $540 \mathrm{~nm}$, and light emitted in NBI is mostly absorbed by hemoglobin, allowing visualization of blood vessels as a dark green color. In regions other than blood vessels, light enters tissue, scatters and reflects, resulting in a brighter appearance.

NBI has recently attracted attention due to its clear visualization of mucosal blood vessels (Fig. 1). Neoplastic vessels tend to proliferate on the surface of cancerous lesions in the digestive 
tract, which makes NBI very useful for qualitative diagnosis of malignant and benign lesions through clear visualization of microvessels (8). In the gynecological field, angiogenesis is also considered as a diagnostic marker of a malignant endometrial lesion $(9,10)$. Therefore, identification of increased angiogenesis or abnormal vessels may play a role in diagnosis of malignant lesions, including those in endometrial carcinoma and its precursors.

To evaluate NBI hysteroscopy for diagnosis of malignant endometrial lesions, we applied NBI in flexible hysteroscopy at our outpatient clinic. Images were placed in 2 groups: those acquired using conventional white light alone and those obtained with white light+NBI. The sensitivity and specificity of diagnoses of atypical endometrial hyperplasia (AEH) or endometrial carcinoma were compared between these groups.

\section{Patients and methods}

Patients and procedures. The subjects were 71 patients with a suspected endometrial lesion in the uterine cavity. These patients underwent flexible hysteroscopy using NBI in addition to conventional white light at the outpatient clinic of our hospital between July 2009 and January 2010, and thereafter were pathologically diagnosed based on surgical specimens. NBI hysteroscopy was performed after obtaining approval from the ethics committee of Keio University School of Medicine and consent from the patients. Patients receiving high-dose medroxyprogesterone acetate (MPA) therapy and those with a history of this therapy were included in the study. Patients with a history of surgery including dilatation and curettage (D\&C) and transcervical resection of the endometrium (TCRE), and those for whom hysterescopy was required, were also included. Patients with major genital hemorrhage, menstruation, possible pregnancy, endometritis, and cervical neoplasia were excluded from hysteroscopy. Pathological diagnoses were made using specimens obtained in surgery, including D\&C, TCRE, and hysterectomy. Based on these diagnoses, the cases were classified into four categories: benign lesion (normal endometrium, endometrial polyp, submucosal myoma, MPA therapy-induced decidual changes), EH, AEH, and endometrial carcinoma.

The instruments used in this study were a $3.1-\mathrm{mm}$ flexible hysterofiberscope (HYF-XP) connected to a CCD digital camera (OTV-S7ProH-HD-12E), a VISERA Pro Xenon light source (CLV-S40Pro) and a VISERA Pro video processor (OTV-S7Pro) (all from Olympus Medical Systems Corp., Tokyo, Japan). An Olympus OEV-191H 19-in high-definition LCD monitor was used and movies were recorded with a DVD recorder (DMRXP12; Panasonic Co., Osaka, Japan).

Hysteroscopy was performed by gynecologists with 5-7 years of experience. Patients were placed in the lithotomy position and the examinations were performed transvaginally without anesthesia. A drip infusion bag was positioned at a level of $\sim 1 \mathrm{~m}$ above the patient to allow a natural drip, and a scope was inserted into the uterine cavity while it was distended. The entire uterine cavity was first observed under white light, followed by switching to NBI by pushing the button on the scope. Lesions were carefully observed using white light and NBI. The frequency of switching between white light and NBI and the duration of observation were not specified.
Evaluation of video images. The series of examinations were video-recorded, and video images were evaluated by 4 raters (none of whom performed hysteroscopy) in the following 5 steps (Fig. 2).

Step 1 (developmental phase I): An expert endoscopist observed all video images, and 6 patients with video images judged to be inappropriate because of poor quality due to a failure to achieve optimal focus, insufficient length of segments and involvement on performance by the 4 raters in the following steps were excluded from the study.

Step 2 (developmental phase II): Video images of 65 patients were edited (a total of 130 videos were prepared) and placed in groups of images acquired with white light alone (WL group) and white light+NBI (NBI group). To eliminate systematic bias in evaluating the videos, the order of evaluation of the images from the two diagnostic tests was block-randomized.

Step 3 (learning phase): To utilize our previously reported hysteroscopic diagnostic criteria for endometrial lesions and atypical vascular images (11) and to standardize the evaluation among the raters, the raters received an explanation of the diagnostic criteria and the features of atypical blood vessels. In the criteria, endometrial lesions are diagnosed taking notice of protruding lesions, necrotizing tissue, and atypical blood vessels. The raters first underwent an interactive training session with images and videos extracted from our digital library of images and videos. An expert endoscopist provided instruction in this session.

Step 4 (demonstration phase): To investigate variation in diagnostic performance among the raters, each rater diagnosed 10 white light video images while blinded to all other clinical information. The accuracy of these diagnoses was compared among the raters.

Step 5 (study phase): Using the 130 block-randomized video images, the raters diagnosed images while blinded to other clinical information. The images were displayed on a large screen, and the each rater simultaneously evaluated the same video image. These videos were independent from those used in the learning and demonstration phases.

Based on the pathological diagnosis as the gold-standard, the sensitivity and specificity of the hysteroscopic diagnoses of $\mathrm{AEH}$ or endometrial carcinoma, requiring treatment using highdose MPA therapy or surgery, were compared between the WL and NBI groups to evaluate the usefulness of NBI hysteroscopy.

Statistical analysis. Descriptive statistics on demographic factors were summarized. The pathological diagnosis of benign lesion (normal, polyp, myoma, decidual change), $\mathrm{EH}, \mathrm{AEH}$, or carcinoma was used as the true disease status. AEH or endometrial carcinoma was defined as disease when calculating the sensitivity. The $95 \%$ confidence intervals (CIs) for sensitivity, specificity and accuracy were estimated with the Clopper-Pearson (exact) method for each rater and diagnostic test. Sensitivities were compared between the diagnostic tests using a McNemar test for each rater. A conditional logistic regression model was used to compare the sensitivities for the diagnostic tests averaged over raters. Agreement between the diagnoses in each test and the pathological diagnosis was evaluated with a weighted $\kappa$ coefficient for each rater. Significance levels in all tests were 5\% (two-sided). All data were analyzed with SAS (version 9.1). 


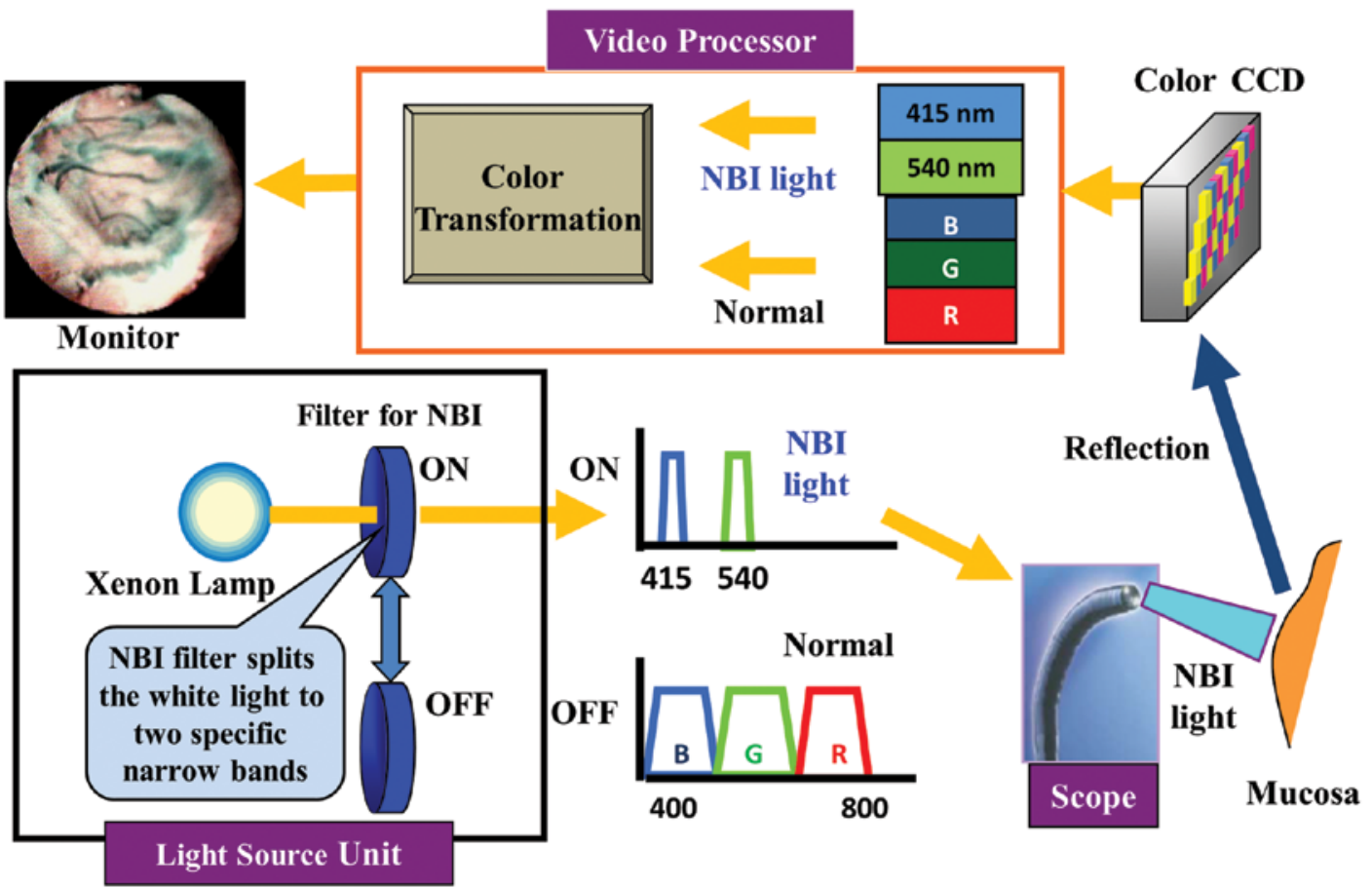

Figure 1. Overview of the NBI system. A filter for NBI is set in front of a xenon lamp in the light source unit and inserted into the light path for NBI observation by pushing a button on the scope. Only 415- and 540-nm narrow-band light passes through the filter, appears as blue light under a scope, and is reflected by the mucosa. Reflected light is caught by a color CCD and the narrow-band images are produced in a video processor and displayed on the monitor.

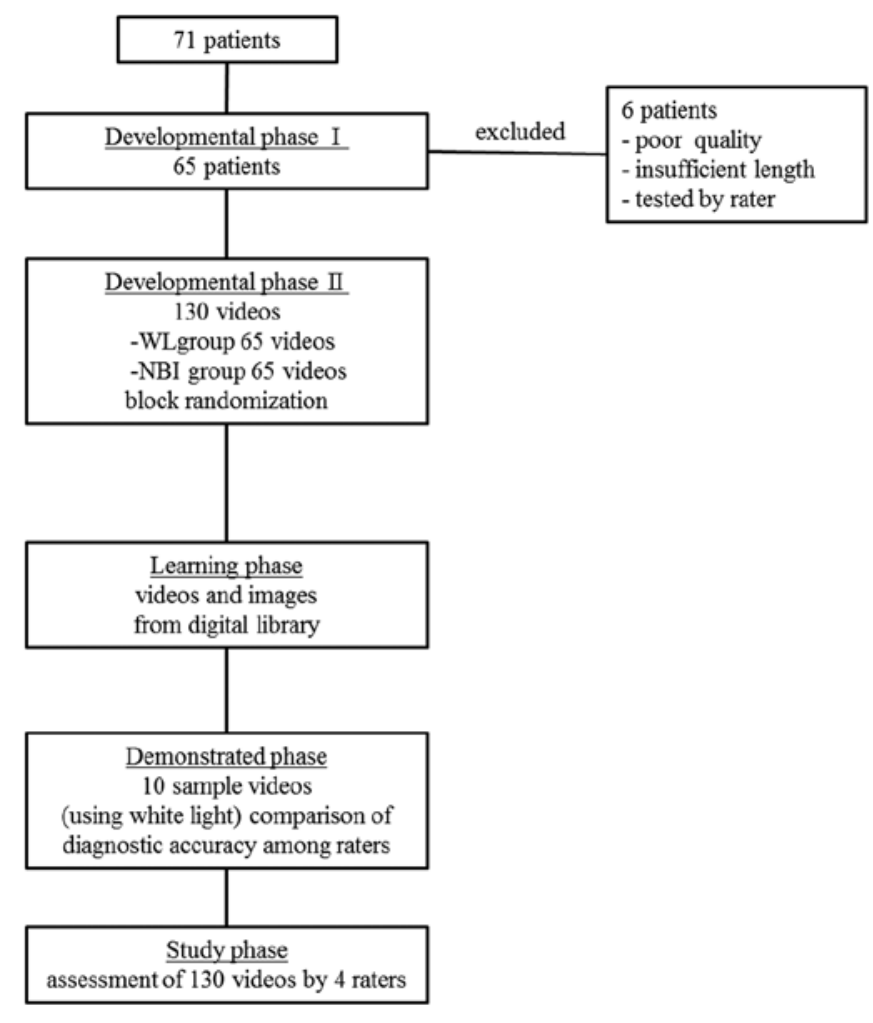

Figure 2. Flow chart of the study design.

\section{Results}

Patient background. All 71 cases were examined by hysteroscopy using white light and NBI without complication, and all

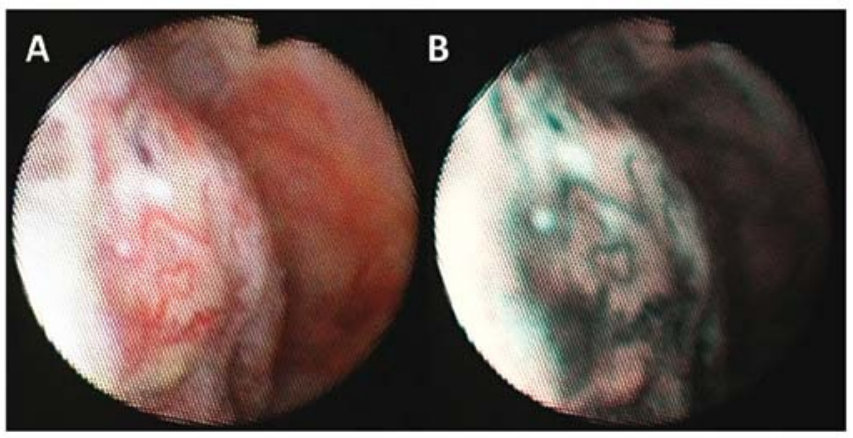

Figure 3. Comparison of visualization of endometrial carcinoma by (A) conventional and (B) NBI hysteroscopy. Atypical blood vessels were found in the protruding lesion area and visualized more clearly by NBI.

had pathological diagnoses of surgical specimens. However, the video images were judged to be inappropriate for evaluation in 6 patients, and therefore analysis was performed for 65 patients. The mean age of the 65 patients was $41.9 \pm 12.5$ years old, and the indications for hysteroscopy were an abnormality on transvaginal ultrasonography or endometrial cytohistology performed at our hospital or elsewhere, judgment of the effect of MPA therapy, metrorrhagia, and hypermenorrhea. The pathological diagnoses were benign lesions in 19 cases (29.2\%; normal endometrium 1, endometrial polyp 6, myoma 4, decidual change 8), $\mathrm{EH}$ in 4 (6.2\%), $\mathrm{AEH}$ in 8 (12.3\%), and carcinoma in $34(65.0 \%)$ (Table I).

Diagnostic performance in demonstration phase among the raters. The 4 raters first judged 10 sample video images acquired under white light [(5 benign lesions [endometrial 
Table I. Patient background.

\begin{tabular}{lc}
\hline Characteristic & Value \\
\hline Age (years) & $41.9 \pm 12.5$ \\
& \\
Indication for hysteroscopy & 22 \\
Referred from other hospitals for abnormality & \\
on ultrasonography or cytohistology & 17 \\
Abnormality on cytology or histological & \\
diagnosis at our hospital & 11 \\
Abnormality on ultrasonography at our hospital & 10 \\
Judgment of MPA therapy & 4 \\
Metrorrhagia & 1 \\
Menorrhagia & \\
Pathological diagnosis & 19 \\
Benign lesion & 1 \\
Normal & 6 \\
Polyp & 44 \\
Myoma & 4 \\
Decidual change & 8 \\
EH & 4 \\
AEH & \\
Carcinoma & \\
\hline
\end{tabular}

MPA, medroxyprogesterone acetate; EH, endometrial hyperplasia; $\mathrm{AEH}$, atypical endometrial hyperplasia.

polyp 3, myoma 1, decidual change 1), $1 \mathrm{EH}, 1 \mathrm{AEH}$, and 3 carcinoma]. Accurate judgments were made for 6 samples by 2 raters and for 7 samples by the other 2 raters, indicating no marked difference in diagnostic accuracy among the raters. The performance of all the raters while blinded to all other clinical information was judged to be satisfactory, compared to historical data collected using white light hysteroscopy (11). Thus, none of the raters were excluded in the following study phase.
Comparison of visualization by white light and NBI hysteroscopy. The 130 videos (2 from each patient) were block-randomized into WL and NBI groups. A comparison of visualization by white light and NBI showed that endometrial blood vessels were more readily observed in images obtained using NBI than in those under white light. In NBI, blood vessels appeared in a dark blue color with enhanced contrast, and consequently atypical blood vessels could be detected easily (Fig. 3).

Sensitivity, specificity and accuracy of white light and NBI groups. The accuracy of the hysteroscopic video imaging diagnoses in the two groups and weighted $\kappa$ coefficients were estimated for each rater based on the pathological diagnosis. Ranges of the accuracy for four raters were $80.0-90.8 \%$ in the NBI group and 64.6-80.0\% in the WL group. With respect to the weighted $\kappa$ coefficients, ranges of the weighted $\kappa$ coefficients for four raters were 0.548-0.806 in the NBI group and 0.379-0.582 in the WL group (Table II). For all raters the hysteroscopic video imaging diagnosis in the NBI group agreed with the pathological diagnosis better than that in the WL group.

The average sensitivities of hysteroscopic diagnosis of the AEH or endometrial carcinoma were $78.6 \%$ in the NBI group and $63.7 \%$ in the WL group. The difference in the average sensitivity between the two groups was significant $(\mathrm{P}<0.001)$. Ranges of the sensitivity for four raters were $69.0-88.1 \%$ in the NBI group and $45.2-73.8 \%$ in the WL group. Ranges of the difference in the sensitivity between the two groups (NBIWL) for four raters were $9.5-23.8 \%$ and the sensitivities in the NBI group were numerically higher than that in the WL group for all of four raters. The average specificities were 92.4 and 93.5\% in the NBI group and in the WL group, respectively. The specificity was comparable between the two groups (Table III).

\section{Discussion}

Development of the NBI system for image-enhanced endoscopy was initiated in May 1999, and Olympus Corp. launched a product in May 2006. Currently, the system is mainly used in digestive endoscopy for the pharynx, esophagus, stomach, and large intestine (12-14). Use of NBI in gynecology has been reported for laparoscopic identification of peritoneal endometriosis $(15,16)$, evaluation of vascular density in endo-

Table II. Accuracy of diagnoses of hysteroscopic video images and weighted $\kappa$ coefficients for each rater.

\begin{tabular}{|c|c|c|c|c|}
\hline \multirow[b]{2}{*}{ Rater } & \multicolumn{2}{|c|}{ Accuracy, \% (95\% CI) } & \multicolumn{2}{|c|}{ Weighted $\kappa$ coefficient $(95 \% \mathrm{CI})$} \\
\hline & WL & NBI & WL & NBI \\
\hline 1 & $64.6(51.8-76.1)$ & $80.0(68.2-88.9)$ & $0.379(0.234-0.523)$ & $0.548(0.402-0.694)$ \\
\hline 2 & $76.9(64.8-86.5)$ & $83.1(71.7-91.2)$ & $0.471(0.315-0.627)$ & $0.654(0.516-0.792)$ \\
\hline 3 & $75.4(63.1-85.2)$ & $80.0(68.2-88.9)$ & $0.456(0.311-0.602)$ & $0.660(0.527-0.794)$ \\
\hline 4 & $80.0(68.2-88.9)$ & $90.8(81.0-96.5)$ & $0.582(0.431-0.733)$ & $0.806(0.682-0.930)$ \\
\hline Average & 74.2 & 83.5 & & \\
\hline
\end{tabular}

WL, white light; NBI, white light+NBI. 
Table III. Sensitivity and specificity of diagnoses of atypical endometrial hyperplasia or endometrial carcinoma in hysteroscopic video images.

\begin{tabular}{|c|c|c|c|c|c|}
\hline \multirow[b]{2}{*}{ Rater } & \multicolumn{2}{|c|}{ Sensitivity, \% (95\% CI) } & \multirow[b]{2}{*}{ P-value ${ }^{a}$} & \multicolumn{2}{|c|}{ Specificity, \% (95\% CI) } \\
\hline & WL & NBI & & WL & NBI \\
\hline \multirow[t]{2}{*}{1} & $19 / 42$ & $29 / 42$ & 0.008 & $23 / 23$ & $23 / 23$ \\
\hline & $45.2(29.8-61.3)$ & $69.0(52.9-82.4)$ & & $100.0(85.2-100.0)$ & $100.0(85.2-100.0)$ \\
\hline \multirow[t]{2}{*}{2} & $31 / 42$ & $35 / 42$ & 0.157 & $19 / 23$ & $19 / 23$ \\
\hline & $73.8(58.0-86.1)$ & $83.3(68.6-93.0)$ & & $82.6(61.2-95.0)$ & $82.6(61.2-95.0)$ \\
\hline \multirow[t]{2}{*}{3} & $27 / 42$ & $31 / 42$ & 0.248 & $22 / 23$ & $21 / 23$ \\
\hline & $64.3(48.0-78.4)$ & $73.8(58.0-86.1)$ & & $95.7(78.1-99.9)$ & $91.3(72.0-98.9)$ \\
\hline \multirow[t]{2}{*}{4} & $30 / 42$ & $37 / 42$ & 0.008 & $22 / 23$ & $22 / 23$ \\
\hline & $71.4(55.4-84.3)$ & $88.1(74.4-96.0)$ & & $95.7(78.1-99.9)$ & $95.7(78.1-99.9)$ \\
\hline \multirow[t]{2}{*}{ Average } & $107 / 168$ & $132 / 168$ & $<0.001$ & $86 / 92$ & $85 / 92$ \\
\hline & 63.7 & 78.6 & & 93.5 & 92.4 \\
\hline
\end{tabular}

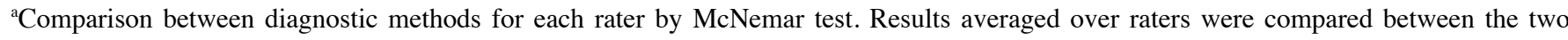
diagnostic methods using a conditional logistic regression model. WL, white light; NBI, white light+NBI.

metriosis (17,18), and diagnosis of endometrial lesions (19-21). In a previous report, we used NBI for flexible hysteroscopy at our outpatient clinic, and compared historical data collected using white light with data collected using white light+NBI. The sensitivities for identification of AEH or endometrial carcinoma were $82.6 \%$ using white light and $97.2 \%$ using white light+NBI, suggesting the usufulness of NBI for diagnosis of endometrial lesions (11). Cicinelli et al also found that the sensitivity of diagnosis of low- and high-risk hyperplasia was significantly higher using NBI compared to white light (19), and Surico et al reported that the sensitivity for diagnosis of endometrial carcinoma and hyperplasia was significantly higher using NBI than white light (20).

Hysteroscopic diagnosis of endometrial lesions is often made with reference to prior clinical information from findings in cytology or histological examination of endometrial tissue, imaging such as transvaginal ultrasonography and MRI, and tumor markers. This information may influence the hysteroscopic diagnosis and account for the lack of standard diagnostic criteria for endometrial lesions using hysteroscopy alone. In this study, raters evaluated video images acquired by hysteroscopy with conventional white light alone and white light+NBI without use of other clinical information. The raters had not been involved in performance of the hysteroscopy. This approach provides a less biased evaluation of the images collected using the NBI system and this study is the first evaluation of NBI hysteroscopy based on visual information (video images) alone.

Hysteroscopic diagnoses are also often made subjectively and may show variation among operators. Thus, to evaluate NBI compared to the conventional method, a study design with greater objectivity is necessary. We performed the current study following the flow chart shown in Fig. 2. First, video images were used without additional clinical information. Second, images were edited and randomized into groups of images acquired using white light alone and those obtained with white light+NBI. To reduce variation of the diagnostic method among the raters and promote objective diagnoses, the raters were given an explanation of our diagnostic criteria for endometrial lesions and atypical vascular images (11), with a sample image display. They also evaluated 10 sample video images acquired using white light alone as a trial, and their diagnostic performance was evaluated to confirm the absence of marked variation. These steps were used to make the comparative study as objective as possible.

The sensitivity for identification of AEH or endometrial carcinoma was higher in the NBI group than in the WL group, indicating that blood vessels can be more readily identified using NBI compared to white light. This effect is because the contrast of mucosal blood vessels is enhanced by NBI, leading to easier detection of endometrial lesions containing atypical blood vessels. The sensitivity of diagnosis in the NBI group was $78.6 \%$, but may be increased in clinical practice with use of additional clinical information. The specificity was similar between the two groups, though it was suggested that NBI enhancement of visualization of blood vessels may also have elevated the false positive rate for detection of lesions. This problem can be reduced by establishment of strict criteria for defining atypical blood vessels. All raters showed increased sensitivity without decreased specificity for images collected using NBI compared to white light, suggesting that any operator can use NBI data after receiving instructions. These results show that NBI hysteroscopy is more useful than current white light hysteroscopy for diagnosis of $\mathrm{AEH}$ or endometrial carcinoma through identification of atypical blood vessels. 
Despite the promising results, the study has some limitations. Since our hospital is a highly advanced medical facility, more patients with AEH or endometrial carcinoma were included in the patient population compared to general hospitals. About 2/3 of the patients had AEH or endometrial carcinoma, and this proportion is higher than those in the studies of NBI hysteroscopy by Cicinelli et al (19) and Surico et al (20). Moreover, the numbers of raters and patients were limited because video records were used, with which a large-scale study cannot be designed.

The high sensitivity of NBI hysteroscopy facilitates judgment of the presence of malignant endometrial lesions, which may be clinically useful for the following reasons. First, biopsy or histological examination of the endometrium may not always be necessary for all patients with a suspected endometrial lesion, reducing unnecessary and painful tests. We use a thin flexible endoscope for NBI hysteroscopy, which is not painful and requires no anesthesia. Second, false-negative results may be obtained in endometrial cytology and histology for endometrial lesions in the uterine cavity, but such lesions can be identified by NBI hysteroscopy. Using hysteroscopy with endometrial cytology and histology will allow localized lesions to be identified, and NBI hysteroscopy increases the diagnostic accuracy and capacity for detection of endometrial lesions. Third, the therapeutic result (presence of residual malignant lesions) of high-dose progesterone therapy can be evaluated by hysteroscopy without performing D\&C.

In conclusion, evaluation of video images without use of other clinical information showed that the sensitivity of NBI hysteroscopy for identifying AEH or endometrial carcinoma is greater than that using conventional white light hysteroscopy. The NBI system for flexible hysteroscopy allows clear visualization of mucosal blood vessels, and may be a useful new imaging tool for supplementing endoscopic diagnosis of malignant endometrial lesions. However, large-scale, multicenter, prospective randomized trials are required to confirm the potential of NBI for endoscopic diagnosis of malignant endometrial lesions.

\section{Acknowledgements}

We thank Takashi Saga, Ayumi Saito, Sayoko Tanaka, Tomoharu Sanada and Kenji Matushima of Olympus Medical Science Sales Co., Ltd. for their technical assistance. We also thank Dr Tai Ohmori of the Department of Surgery, Keio University School of Medicine for advice on the NBI system. This study was supported by the Japanese Foundation for Research and Promotion of Endoscopy (JFE) Grant, the Japan Society for the Promotion of Science (JSPS) through a Grant-in-Aid for Scientific Research (KAKENHI), a Grant-in Aid for Scientific Research (C) (22591866), the Takeda Science Foundation, and the Foundation for Promotion of Cancer Research.

\section{References}

1. Balasch J: Investigation of the infertile couples: investigation of the infertile couple in the era of assisted reproductive technology: a time for reappraisal. Hum Reprod 15: 2251-2257, 2000.

2. Brand A: Diagnosis of endometrial cancer in women with abnormal uterine bleeding. J SOGC 86: 1-3, 2000.

3. Chanbers JT and Chanbers SK: Endometrial sampling: When? Where? Why? With what? Clin Obstet Gynecol 35: 28-39, 1992.

4. Creasman WT: Endometrial cancer: incidence, prognostic factors, diagnosis, and treatment. Semin Oncol 24: S1-140-S1-50, 1997.

5. Garuti G, Sambruni I, Colonnelli M and Luerti M: Accuracy of hysteroscopy in predicting histopathology of endometrium in 1500 women. J Am Assoc Gynecol Laparosc 8: 207-213, 2001.

6. Lasmar RB, Barrozo PR, De Oliveira MA, Coutinho ES and Dias R: Validation of hysteroscopic view in cases of endometrial hyperplasia and cancer in patients with abnormal uterine bleeding. J Minim Invasive Gynecol 13: 409-412, 2006.

7. Gono K, Obi T, Yamaguchi M, et al: Appearance of enhanced tissue features in narrow-band endoscopic imaging. J Biomed Opt 9: 568-577, 2004.

8. Muto M, Katada C, Sano Y and Yoshida S: Narrow band imaging: a new diagnostic approach to visualize angiogenesis in superficial neoplasia. Clin Gastroenterol Hepatol 3: 16-20, 2005.

9. Abukafia O, Triest WE and Sherer DM: Angiogenesis in malignancies of the female genital tract. Gynecol Oncol 72: 220-231, 1999.

10. Stefansson IM, Salvesen HB and Akslen LA: Vascular proliferation is important for clinical progress of endometrial cancer. Cancer Res 66: 3303-3309, 2006

11. Kisu I, Banno K, Kobayashi Y, et al: Flexible hysteroscopy with narrow band imaging (NBI) for endoscopic diagnosis of malignant endometrial lesions. Int J Oncol 38: 613-618, 2011.

12. Muto M, Saito Y, Ohmori T, Kaise M, Inoue $\mathrm{H}$ and Ishikawa $\mathrm{H}$ : Multicenter prospective randomized controlled study on the detection and diagnosisi of superficial squamous cell carcinoma by back-to-back endoscopic examination of narrowband imaging and white light observation. Gastrointest Endosc 65: AB110, 2007.

13. Sano Y and Horimatsu T: Magnifying observation of microvascular architecture of colorectal lesions using narrow-band imaging system. Dig Endosc 18: 44-51, 2006.

14. Muto M and Horimatsu T: Narrow-band imaging of the gastrointestinal tract. J Gastroenterol 44: 13-25, 2009.

15. Barrueto FF and Audlin KM: The use of narrowband imaging for identification of endometriosis. J Minim Invasive Gynecol 15: 636-639, 2008

16. Farrugia M, Nair $M$ and Kontronis: Narrow band imaging in endometriosis. J Minim Invasive Gynecol 14: 393-394, 2007.

17. Kuroda K, Kitade M, Kikuchi I, et al: Vascular density of peritoneal endometriosis using narrow-band imaging system and vascular analysis software. J Minim Invasive Gynecol 16: 618-621, 2009.

18. Kuroda K, Kitade M, Kikuchi I, et al: Peritoneal vascular density assessment using narrow-band imaging and vascular analysis software, and cytokine analysis in women with and without endometriosis. J Minim Invasive Gynecol 17: 21-25, 2010.

19. Cicinelli E, Tinelli R, Colafiqlio G, et al: Reliability of narrowband imaging (NBI) hysteroscopy: a comparative study. Fertil Steril 94: 2303-2307, 2010.

20. Surico D, Vigone A, Bonvini D, Tinelli R, Leo L and Surico N: Narrow-band imaging in diagnosis of endometrial cancer and hyperplasia: a new option? J Minim Invasive Gynecol 17: 620-625, 2010.

21. Surico D, Viqone A and Leo L: Narrow band imaging in endometrial lesions. J Minim Invasive Gynecol 16: 9-10, 2009. 